\title{
Universidade e conectividade: conhecimentos em interação com a comunidade
}

\section{University and connectivity: knowledge in interaction with the community}

Article Info:

Article history: Received 2021-03-29 / Accepted 2021-03-29 / Available online 2021-03-30

doi: 10.18540/jcecvl7iss1pp12230-01-06e

Tarciso Binoti Simas

ORCID: https://orcid.org/0000-0002-1687-7582

Universidade Federal do Sul e Sudeste do Pará, Brasil

E-mail: tarcisobinoti@gmail.com

Carlos Maviael de Carvalho

ORCID: https://orcid.org/0000-0002-7777-4659

Universidade Federal do Sul e Sudeste do Pará, Brasil

E-mail: maviael.mcarvalho@gmail.com

Samuel da Silva Sousa

ORCID: https://orcid.org/0000-0001-8947-3338

Universidade Federal do Sul e Sudeste do Pará, Brasil

E-mail: samueldasilvasousa090@gmail.com

Ivan Carlos Franco

https://orcid.org/0000-0003-4811-8158

Centro Universitário da FEI, Brazil

E-mail: icfranco@gmail.com

Wagner dos Reis Marques Araújo

https://orcid.org/0000-0003-0793-0043

Universidade do Estado de Minas Gerais, UEMG - Unidade Abaeté

E-mail: marquesreis@hotmail.com

Antonio Marcos de Oliveira Siqueira

http://orcid.org/0000-0001-9334-0394

Universidade Federal de Viçosa, UFV, Brazil

E-mail: antonio.siqueira@ufv.br

\section{Resumo}

O Conara - Congresso Araguaiense de Ciências Exata, Tecnológica e Social Aplicada - é um evento anual realizado pelos servidores do Instituto de Engenharia do Araguaia da Universidade Federal do Sul e Sudeste do Pará - IEA/Unifesspa. O evento Conara tem como objetivos contribuir e divulgar a produção acadêmico-científica e profissional inerentes à formação, à pesquisa, à extensão e ao desenvolvimento cultural e tecnológico nas áreas de Arquitetura e Urbanismo, Engenharia Civil, Licenciatura em Matemática, Gestão de Segurança e Meio Ambiente e campos afins. Nessa formação tríade com cursos de distintas naturezas, o IEA/Unifesspa realizou de forma gratuita o primeiro Conara em 2019. Com as restrições causadas pela Covid-19, a $2^{\mathrm{a}}$ edição deste congresso gratuito e acessível foi realizada de forma remota em 2020 com tema "Universidade e conectividade: conhecimentos em interação com a comunidade". Essa conexão virtual imposta pelo distanciamento social se tornou uma oportunidade para que esse congresso local ganhasse proporções maiores. Um dos principais ganhos foi a parceria com este periódico, The Journal of Engineering and Exact Sciences - jCEC, onde os melhores trabalhos do Conara foram publicados. Isso repercutiu no aumento de participantes e de trabalhos submetidos no evento em relação a $1^{\mathrm{a}}$ edição. Durante os cinco dias do II Conara, entre 23 e 27 de novembro, foi possível receber participantes de diversas partes do Brasil e do exterior através de uma programação de palestras, minicursos e sessões temáticas. Assim, a realização deste evento permite que, mesmo em meio aos desafios, o 
IEA/Unifesspa siga seus propósitos de interiorização do ensino superior público, gratuito e de qualidade e de discussão científica acessível e conectada com a comunidade local e global.

Palavras-chave: Universidade Pública. Conectividade. Interiorização do ensino superior.

\begin{abstract}
Conara - Araguaiense Congress of Exact, Technological and Applied Social Sciences - is an annual event held by the Araguaia Engineering Institute of the Federal University of the South and Southeast of Pará - IEA / Unifesspa. The Conara event aims to contribute and disseminate academicscientific and professional production inherent to training, research, extension and cultural and technological development in the areas of Architecture and Urbanism, Civil Engineering, Degree in Mathematics, Security and Environment Management Environment and related fields. In this triad formation with courses of different natures, the Institute held for free the first Araguaia Congress of Exact, Technological and Applied Social Sciences (Conara) in 2019. With the restrictions caused by Covid-19, the 2 nd edition of this free and accessible congress was held remotely in 2020 with the theme "University and connectivity: knowledge in interaction with the community". This virtual connection imposed by social distance became an opportunity for this local congress to gain greater proportions. One of the main gains was the partnership with The Journal of Engineering and Exact Sciences - jCEC, where Conara's best works are published. This had an impact on the increase in the number of participants and papers submitted to the event in relation to the 1st edition. During the five days of II Conara, between 23 and 27 November, it was possible to receive participants from different parts of Brazil and abroad through a program of lectures, short courses and thematic sessions. Thus, the realization of this event allows, even in the midst of challenges, the IEA/Unifesspa to follow its purposes of internalizing public, free and quality higher education and accessible and connected scientific discussion with the local and global community.
\end{abstract}

Keywords: Public university. Connectivity. Higher education interiorization.

\title{
1. Introdução
}

A formação acadêmica e profissional consolidada de um indivíduo perpassa o espaço físico destinado para ministração de conteúdos. Trata-se de um processo de busca contínua por novos conhecimentos que exige envolvimento do acadêmico nas diversas atividades propostas pelas instituições no âmbito do ensino, da pesquisa e da extensão. Como bem destacam Arboit e Santiago (2011):

Os eventos científicos são considerados meios mais informais e, com efeito, mais ágeis na transmissão e troca do conhecimento científico. Diferentemente dos meios convencionais de comunicação, tais como livros e periódicos, grande parte da informação é transferida oralmente, estimulando o debate instantâneo dos especialistas interessados no tema.

Além da interação acadêmica como destacam Pinheiro, Silva e Siqueira (2017), os eventos científicos permitem "o vislumbre de parcerias para futuros projetos e colaborações, tanto na formação de recursos humanos, como no desenvolvimento de projetos de pesquisa".

Nesse sentindo, os eventos científicos constituem importantes ferramentas para os discentes divulgarem suas pesquisas, para trocarem experiencias e conhecimentos adquiridos em suas vivencias universitárias, para a expansão da capacidade intelectual, para o crescimento pessoal e para a atualização de saberes (SILVA et al., 2019). Figueredo et al. (2016) destaca que os estudantes precisam ter maior envolvimento nessas atividades, uma vez que complementará às atividades acadêmicas e ao conhecimento adquirido em sala de aula.

É com esse intuito que o Instituto de Engenharia do Araguaia da Universidade Federal do sul e Sudeste (IEA/Unifesspa) busca desenvolver eventos científicos que promovam a troca de 
experiencias e informações, de maneira a agregar conhecimentos e valores para formação profissional. Essa Unidade Acadêmica da Unifesspa, em Santana do Araguaia, Pará, iniciou suas atividades em 2014, com o curso de Licenciatura em Matemática. Com essa composição, realizou nos anos de 2015 e 2017, o I e o II Encontro Araguaiense de Matemática, com palestras, mesasredondas e minicursos. Com a oferta dos cursos, em 2018, de graduação em Engenharia Civil e, em 2019, de graduação em Arquitetura e Urbanismo, o IEA lançou-se ao desafio da realização de forma gratuita do I Congresso Araguaiense de Ciências Exata, Tecnológica e Social Aplicada (Conara), em 2019, com tema central "Problematizando, projetando e construindo para um desenvolvimento mais sustentável”, graças ao financiamento da Fundação Amparo e Desenvolvimento da Pesquisa Fadespa através da Chamada $n^{0}$ 001/2019 - Apoio à Realização de Eventos Científicos, Tecnológicos e de Inovação. Foram realizadas diversas atividades, envolvendo exposições, comunicação oral, apresentação de pôster, publicação de artigos completos e resumos em anais, palestras, mesa redonda, oficinas, minicursos e visitas técnicas. Essa $1^{\circ}$ edição aconteceu no período de 18 a 23 de novembro de 2019, com uma programação diversificada que incluiu 10 minicursos, 13 oficinas, 7 palestras, 1 exposição de pôsteres, 1 amostra de documentário, 2 visitas técnicas, 2 mesa-redonda e 1 feira de física. Além disso, o evento contou a participação de profissionais de várias instituições que não hesitaram em compartilhar conhecimento com os congressistas.

Com base na experiência bem sucedida da primeira edição, o Conara se tornou um evento anual de culminância de atividades de ensino, pesquisa e extensão; de divulgação dos cursos do IEA/Unifesspa para a comunidade araguaiense; de interação de conhecimentos entre docentespesquisadores e estudantes de graduação, entre docentes e estudantes da educação superior e docentes e estudantes da educação básica, entre comunidade universitária e interessados na produção de conhecimento que estão na área de abrangência dos cursos do IEA/Unifesspa. Trata-se de um evento gratuito realizado pelos servidores do IEA/Unifesspa.

Em 2020, a pandemia da Covid-19 nos impôs diversos desafios. Apesar dos impactos negativos, o desafio de realizar um congresso de forma remota por conta do distanciamento social abriu novas oportunidades. Este congresso local ganhou novas e maiores proporções em sua segunda edição, tal como a parceria com The Journal of Engineering and Exact Sciences - jCEC, onde os melhores trabalhos do Conara foram publicados. O tema central escolhido foi "Universidade e Comunidade: conhecimentos em interação" buscando a conceção entre os conhecimentos científicos das áreas a que estão relacionados os cursos do IEA/Unifesspa com os saberes comunitários. Nessa perspectiva, esse artigo busca relatar a realização do II Conara realizado entre 23 e 27 de novembro de 2020 pelo IEA/Unifesspa, com enfoque nos desafios superados e avanços alcançados através do evento.

\section{Sobre o II Conara}

Um dos maiores desafios para realização do II Conara foi a pandemia da Covid-19 que não permitiu a realização do evento presencial como estava programado. Dessa maneira, o congresso teve que se adaptar a um novo formato virtual com a criação do canal do IEA no YouTube, conforme se observa na Figura 1. A temática escolhida para o evento "Universidade e conectividade: conhecimentos em interação com a comunidade" diz respeito ao momento que estamos vivenciando de inteira conectividade com o mundo, onde a Universidade se mostra fundamental para a promoção de um diálogo em rede com sua comunidade acadêmica e a população local.

O II Conara foi realizado entre os dias 23 e 27 de novembro de 2020. Durante esses cinco dias de evento (Quadro 1), por meio de palestras, sessões temáticas e minicursos, o II Conara buscou trazer à tona a produção de conhecimento relacionada às mais variadas formas de interação com a sociedade, os discentes e profissionais da região, que fortalecem a pesquisa e extensão, bem como o desenvolvimento de métodos e práticas que promovem esse dinamismo presente entre a Universidade e a comunidade. 


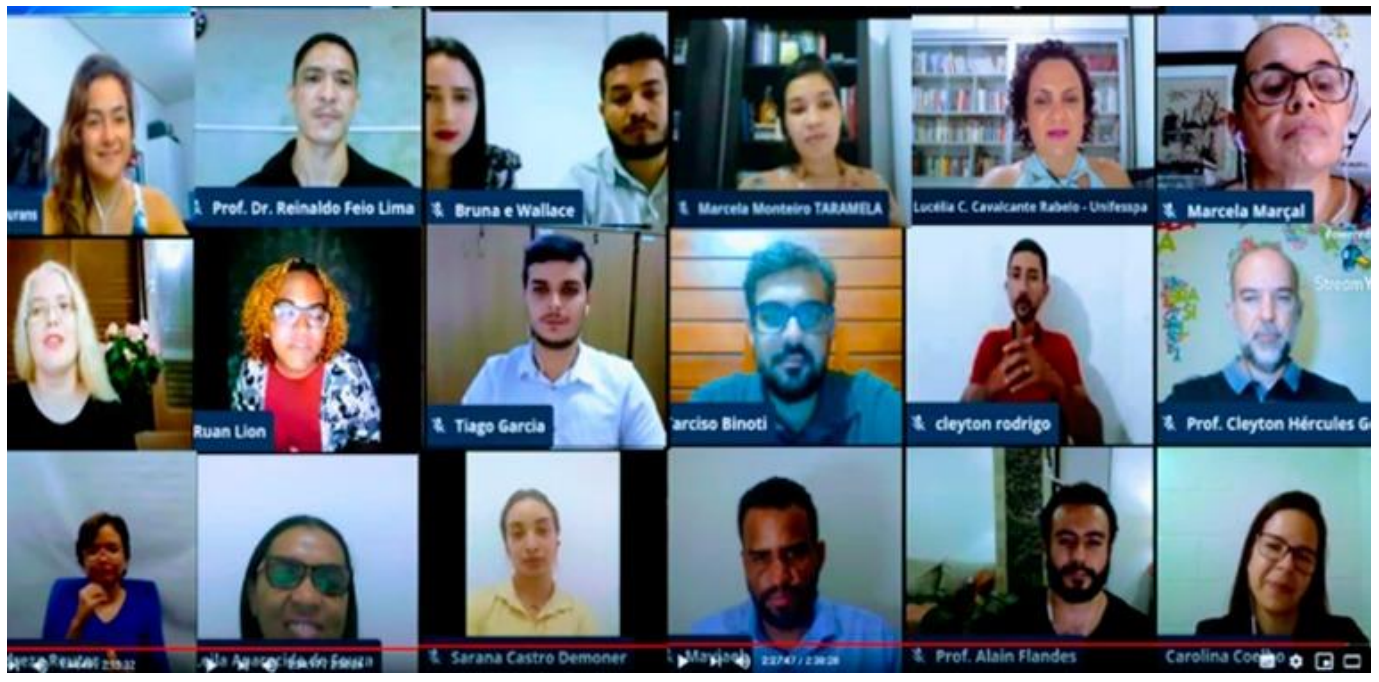

Figura 1 - Transmissão do II Conara via plataforma do YouTube.

Fonte: Autores (2021).

\section{Quadro1 - Cronograma da Programação do II Conara}

\begin{tabular}{|c|c|}
\hline \multicolumn{2}{|r|}{ Segunda-feira } \\
\hline Horário & Atividade \\
\hline 10:00 às 11:40 & Sessão especial: edificações do campus do instituto de engenharia do Araguaia \\
\hline 14:00 às $18: 00$ & Minicurso: bancos de dados aplicados $1^{\circ}$ encontro: \\
\hline 14:00 às 17:00 & Sessão temática: Arquitetura e Urbanismo \\
\hline $15: 10$ às $16: 50$ & Sessão temática: Engenharia Civil e Gestão de segurança e Meio Ambiente \\
\hline 19:00 ás 20:40 & $\begin{array}{l}\text { Solenidade de abertura: universidade e conectividade: conhecimentos em interação com a } \\
\text { comunidade; } \\
\checkmark \quad \text { Tema1: Escuela para la equidad: processo projetual de acordos entre academia e } \\
\quad \text { comunidade rural no México; } \\
\checkmark \quad \text { Tema 2: A importância das ações sociais na formação do engenheiro humano-Casos } \\
\quad \text { no Camtuc-UFPA; } \\
\checkmark \quad \text { Tema 3: Ensino, pesquisa e extensão em parceria com a comunidade. }\end{array}$ \\
\hline \multicolumn{2}{|r|}{ Terça-feira } \\
\hline 10:00 às 11:40 & $\begin{array}{l}\text { Tema Palestra 3: Tecnologias digitais contemporâneas aplicadas ao processo criativo de } \\
\text { projeto }\end{array}$ \\
\hline $14: 00$ às $17: 00$ & Sessão temática: Arquitetura e Urbanismo \\
\hline $14: 30$ às $17: 30$ & Sessão temática: Engenharia Civil e Gestão de segurança e Meio Ambiente \\
\hline 19:00 às 20:40 & $\begin{array}{cl}\text { Tema Palestra 4: ATHIS - Assistência Técnica de Habilitação de Interesse Social } \\
\\
\checkmark & \text { Tema 01: ATHIS - Assistência Técnica de Habitação de Interesse Social; } \\
\checkmark & \text { Tema 02: Ensino prático e Assistência Técnica Habitacional; } \\
\checkmark & \text { Tema 03: Assessoria pelo direito à cidade: atuação profissional da Taramela. }\end{array}$ \\
\hline \multicolumn{2}{|r|}{ Quarta-feira } \\
\hline 10:00 às 11:40 & Tema Palestra 5: Análise e previsão de tempo meteorológico para cronograma de obras \\
\hline 14:00 às 17:00 & Sessão temática: Arquitetura e Urbanismo \\
\hline $14: 30$ às $17: 50$ & Sessão temática: Engenharia Civil e Gestão de segurança e Meio Ambiente \\
\hline $14: 30$ às $17: 50$ & Sessão temática: Licenciatura em Matemática \\
\hline
\end{tabular}




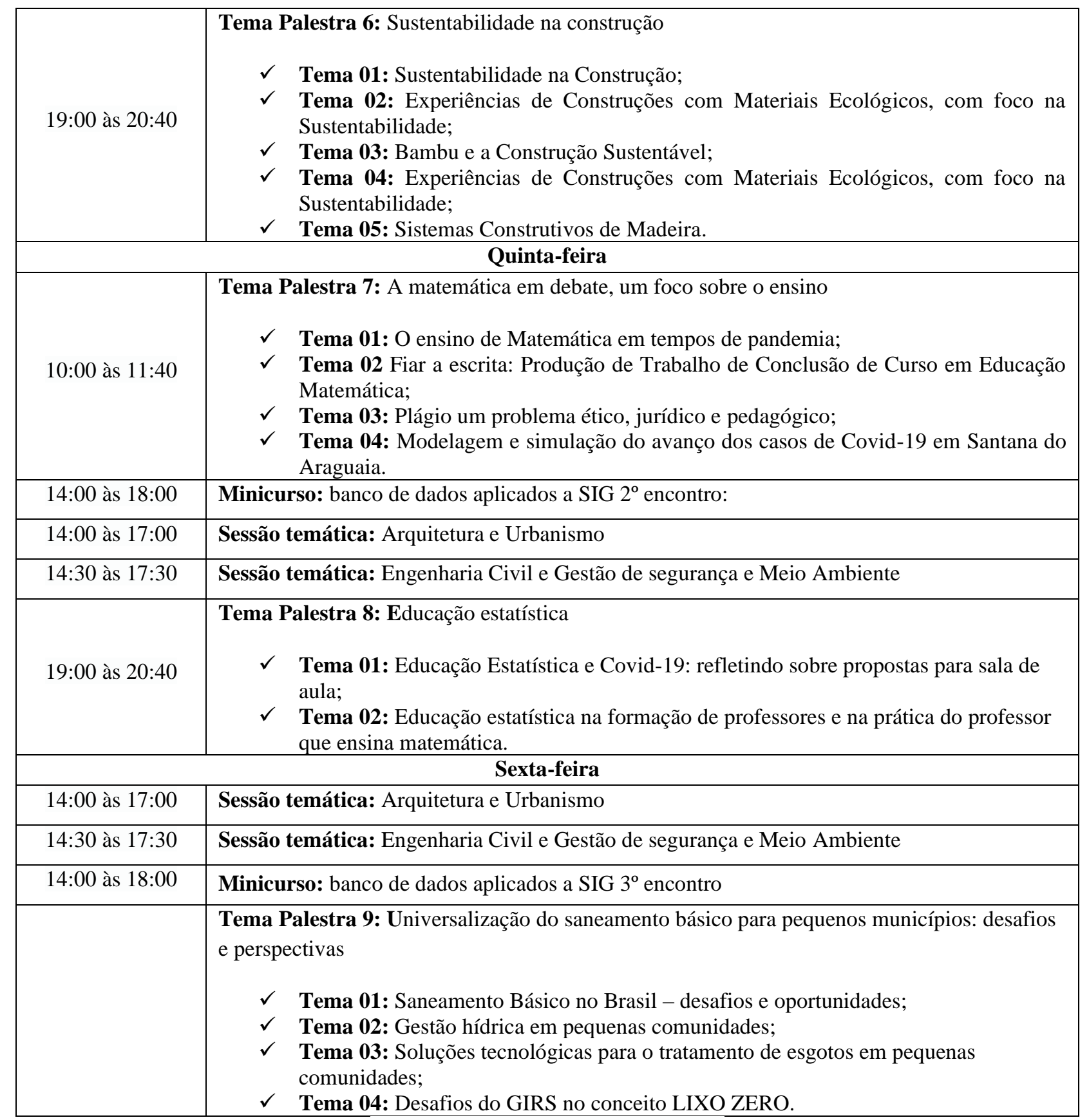

Elaboração pelos autores (2021).

É válido enfatizar que os desafios impostos pela atualidade não foram barreiras para a realização do congresso. Foi realizada a parceria com esta revista, The Journal of Engineering and Exact Sciences - jCEC, que publica os melhores trabalhos do II Conara em edição especial. Com isso, da primeira para a segunda edição, observa-se um aumento no número de inscritos, na composição da comissão organizadora e no quantitativo de artigos submetidos. Outro ponto positivo da II edição do Conara diz respeito ao alcance do evento que obteve proporções maiores, com a participação de congressistas de 24 Instituições de ensino, a saber: Universitat de Barcelona, Universidade Federal do Sul e Sudeste do Pará, Faculdade Ideal, Universidade Federal do Pará, Universidade Federal de Sergipe, Instituto Federal do Pará, Universidade Federal de Pernambuco, Instituto Federal de Alagoas, Centro Universitário de João Pessoa, Instituto Federal da Paraíba, Universidade de Brasília, Universidade Federal de Mato Grosso, Universidade Estadual de Goiás, Universidade Federal do Rio de Janeiro, Universidade de São Paulo, Centro Universitário Luterano de Palmas, Centro Universitário Pitágoras, Universidade Estadual de Londrina, Universidade Maurício de Nassau, Universidade Federal de Rondônia, Universidade Federal do Amazonas, 
Universidade Federal da Bahia, Centro Federal de Educação Tecnológica de Minas Gerais, Universidade Federal de Minas Gerais e Faculdade Facimp Wyden em Imperatriz.

Assim, a realização de um evento dessa magnitude mostra que, apesar dos desafios da interiorização do ensino superior público, o IEA/Unifesspa vem buscando desenvolver seu papel na formação superior de cidadãos.

\section{Considerações}

A Universidade é um espaço de debate e de formação de conhecimentos que auxiliam na formação acadêmica e profissional de milhares de pessoas no mundo inteiro. Nessa perspectiva, os eventos científicos surgem como fator primordial para auxiliar os estudantes em seu desenvolvimento acadêmico e profissional, possibilitando o contato com a realidade vivenciada por outros profissionais, práticas e conhecimentos. Nessa busca, o IEA/Unifesspa vem realizando anualmente o Conara. Em 2020, frente ao cenário de insegurança sanitária da disseminação do vírus da Covid-19, o IEA/Unifesspa não deixou de cumprir sua missão, adaptando a realização do II Conara de evento presencial para virtual, mantendo seu caráter gratuito e acessível. Apesar dos desafios, evidencia que o evento apresenta avanços positivos referente ao número de membros da comissão organizadora, ao número de artigos submetidos, ao número de inscritos e ao número de instituições que participaram do evento em relação ao I Conara. Além dos números, o II Conara cresce em qualidade com novas parcerias e uma programação ampla com a participação de importantes profissionais.

\section{Agradecimentos}

Os autores agradecem o apoio por parte do Instituto de Engenharia do Araguaia da Universidade Federal do Sul e Sudeste do Pará - IEA/Unifesspa e da Universidade Federal de Viçosa (UFV). O presente trabalho foi realizado com apoio da Coordenação de Aperfeiçoamento de Pessoal de Nível Superior - Brasil (CAPES) - Código de Financiamento 001.

\section{Referências}

Arboit, Aline Elis, \& Bufrem, Leilah Santiago. (2011). The production of scientific research in national events in the field of information science. Transinformação, 23(3), 207217. https://doi.org/10.1590/S0103-37862011000300003

Figueredo, L. L., Aquino, A. F. C., Andrade, E. N., Rosas, L. S. (2016). A importância da participação dos estudantes do ensino superior em eventos científicos para sua formação acadêmica. Anais III Congresso Nacional de Educação, Campina Grande. Disponível em: <https://www.editorarealize.com.br/index.php/artigo/visualizar/19884>. Acesso em: 29/03/2021.

Pinheiro, C. L., Silva, G. F. da, \& Siqueira, A. M. de O. (2017). AVALIAÇÃO DO EVENTO CIENTÍFICO: SEMANA ACADÊMICA DE ENGENHARIA QUÍMICA - SAEQ 2016. The Journal of Engineering and Exact Sciences, 3(4), 0595-0602. https://doi.org/10.18540/jcecvl3iss4pp0595-0602

Silva, E. V., Giongo, C. L. M., Rocha, V. M. Possatti (2019). Importância da participação em eventos científicos para a formação em fisioterapia: relato de experiência. Cadernos de educação, saúde e fisioterapia, 6 (12). http://revista.redeunida.org.br/ojs/index.php/cadernos-educacao-saudefisioter/article/view/2855 